\section{KV-SafeNet: DGN tauscht alte Zugangsgeräte aus}

Der Telematik-Dienstleister „Deutsches Gesundheitsnetz Service GmbH“ (DGN) versorgt seine $\mathrm{KV}$-SafeNet-Kunden der ersten Stunde derzeit mit neuen Zugangsgeräten. Wie das Unternehmen meldet, sei der vorkonfigurierte Router "AVM FRITZ!Box", den es seit zwei Jahren raus aus dem Sortiment habe, nicht mehr für die Teilnahme am sicheren KVNetz zugelassen. Der Grund: eine neue KV-SafeNet-Richtlinie. Den rund 1.150 betroffenen Kunden wird der Umstieg auf das zertifizierte SafeNet-Zugangsgerät "DGN GUSbox S" angeboten. Obwohl die GUSbox als Mietgerät zur Verfügung gestellt wird, sollen betroffenen Praxen während der Vertragslaufzeit keine Mehrkosten entstehen.

\section{Microsoft stellt E-Mail-Dienst um}

Microsoft erneuert seine Web-MailDienste: Alle bisherigen Hotmail-Nutzer werden demnächst auf das neue Angebot Outlook.com umgestellt. Outlook. com bietet unter anderem die Einbindung von Twitter, Facebook, Linkedln und Google direkt im Posteingang. So können etwa aktuelle Twitter- oder Facebook-Einträge von Bekannten eingeblendet werden, während man ihre Mails liest. Zudem sind die Web-Apps der Office-Büroprogramme wie Word, Excel und PowerPoint integriert. Bei Microsofts Speicherdienst SkyDrive gibt es dazu 7 Gigabyte Platz gratis. Das "Upgrade“ auf Outlook.com werde für die Hotmail-Nutzer reibungslos verlaufen, versprach $\mathrm{Mi}$ crosoft. Die bisherige Mail-Adresse bleibe genauso erhalten wie alle Ordner und Einstellungen. Bis Sommer soll der Wechsel aller Hotmail-Nutzer zum neuen Dienst abgeschlossen sein.

\section{Update für den Mozilla-Browser Firefox}

Mozilla hat seinen Internet-Browser Firefox überarbeitet. Die neue Version für Windows, Mac und Linux verfügt über einen integrierten PDF-Viewer. Das ist deshalb interessant, weil sich PDF-Dateien nun direkt im Browser lesen lassen, ohne dass Nutzer Plugins installieren oder die Datei-Inhalte herunterladen müssen. Außerdem steht die AndroidVersion des Firefox nun für mehr Smartphones mit ARMv6-Prozessor zur Verfügung (z.B. LG Optimus One, T-Mobile myTouch 3G Slide, HTC Wildfire S, ZTE R750). vektorbasierte Zeichenprogramme. Diese sind allerdings eher etwas für geübtere PC-Nutzer. Wer erst einmal testen will, ob er mit der Logogestaltung zurecht kommt, kann das mit dem kostenfreien Programm Inkscape (http://inkscape.org/) tun, das Bitmaps vektorisieren kann und über eine Vielfalt von Filtern und Spezialeffekten verfügt.

Wer gleich in ein großes Layoutprogramm investieren will, sollte sich unbedingt vorab die Frage stellen: Bin ich eher Layouteinsteiger oder geübter PCAnwender? Für Einsteiger eignen sich nämlich auch hier vorrangig Programme, die ein breites Angebot an Mustern und Vorlagen für Flyer, Poster, Broschüren und Newsletter im Gepäck haben, die sich schnell auf die individuellen Bedürfnisse der Praxis anpassen lassen. Wer wie gesagt auch sein Praxislogo selbst gestalten will, sollte sich eine Software suchen, die entsprechende Tools bietet. Hier wäre auch eine Option zum Freizeichnen wichtig - allerdings mit überschaubarer Menüführung.

\section{Woher kommen schöne Fotos?}

Damit es hinterher mit dem Druck entweder über den Praxisdrucker, im Copyshop oder gar in der Druckerei klappt - sollte es für Flyer, Broschüren etc. Vorlagen in den gängigen Seitengrößen geben. Und es sollten sich Druckdateien, also PDF, direkt aus der Anwendung heraus erstellen lassen. Ein gutes Programm für Einsteiger ist „Magix Page \& Layout Designer 2013“, das in der Vollversion um die $120 €$ kostet.
Was man auch wissen muss: Die Programme beinhalten oft keine Möglichkeit der Fotobearbeitung. Das heißt, hierfür wird eventuell ein zusätzliches Programm benötigt. Für kleinere Projekte reichen häufig die mitgelieferten Bearbeitungsprogramme der eigenen Digitalkamera aus. Oder aber Praxen nutzen Schmuckbilder professioneller Bildagentur wie fotolia (http://de.fotolia. com/), hier muss - außer dem Bildzuschnitt - wenig an den Fotos nachgearbeitet werden. Wer professioneller Fotos bearbeiten will, benötigt hingegen eine Bildretusche- und montage-Software. Der Klassiker ist hier „Photoshop“. Aber auch „Corel Photo.Paint" hat sich einen Namen gemacht. Und es gibt mit Gimp (http://www.gimp.org/) durchaus eine gute Freeware-Alternative.

Für das Erstellen von Grafiken braucht es bei einigen Layoutprogrammen ebenfalls eine Zusatz-Software. Ein Programm mit vielen Möglichkeiten ist hier „Illustrator“ - allerdings benötigt die Handhabung etwas Übung.

Praxisteams, die gestalterisch frei sein und so richtig loslegen wollen, können sich auch an dem echten Design-Klassiker „InDesign“ versuchen. Um die Möglichkeiten des Programms auszutesten und zu lernen, braucht es allerdings Zeit. Dann aber lassen sich Broschüren völlig frei nach eigenem Gusto gestalten.

$\mathrm{Zu}$ nahezu allen Layoutprogrammen gibt es Testversionen. Daher der Rat: Gerade vor größeren Software-Investitionen das Programm vorab auf Herz und Nieren testen.

RebekkaHöhl

\title{
Ein „Social Portal“ für Gesundheitsdaten
}

Die Stephanus IT GmbH, ein Anbieter für ITK-Infrastrukturen und Lösungen im Klinik- und Pflegesektor, hat ein „Social Portal“ für eine integrierte Gesundheitsdatenverwaltung aufgelegt. Es handele sich um eine cloudbasierte Lösung, auf die das Fachpersonal und Patienten via Browser zugreifen könnten. Es soll aber ebenso möglich sein, über eine USB- oder Bluetooth-Anbindung Daten von medizinischen Messgeräten direkt an die Patientenakte im „Social Portal“ zu übertragen. Alle Daten werden dabei verschlüsselt an das Rechenzentrum und vom Rechenzentrum, in dem die Datenserver stehen, übertragen. Zudem können laut der Stephanus IT GmbH die Patienten selbstbestimmt festlegen, wer auf die personenbezogenen Daten zugreifen darf.

http://stephanus.qa2.grcdemo.com/ 